\title{
The Current State of the Pediatric Emergency Medicine Workforce and Innovations to Improve Pediatric Care
}

Gregory W. Faris, MD ${ }^{1}$, James P. Marcin, MD, MPH², Elizabeth Weinstein, MD³

${ }^{1}$ Assistant Clinical Professor, Departments of Emergency Medicine and Pediatrics, Indiana University School of Medicine, Indianapolis EMS, Indianapolis, IN; P: 317880-3900 F: 317-880-0545; gfaris@iu.edu

2 Professor, Department of Pediatrics, UC Davis Children's Hospital, Sacramento, CA; P: 916-734-4726; F: 916-456-2235; jpmarcin@ucdaviws.edu

${ }^{3}$ Associate Clinical Professor, Departments of Emergency Medicine and Pediatrics, Indiana University School of Medicine, Indianapolis EMS, Indianapolis, IN; P: $317-$ 880-3900 F: 317-880-0545; elweinst@iu.edu

Grant Support: None

Corresponding Author and Reprint Requests:

Gregory W. Faris; 720 Eskenazi Avenue FT, $3^{\text {rd }}$ Floor Department of Emergency Medicine Indianapolis, IN 46202; gfaris@iu.edu

This is the author's manuscript of the article published in final edited form as:

Faris, G. W., Marcin, J. P., \& Weinstein, E. (2018). The Current State of the Pediatric Emergency Medicine Workforce and Innovations to Improve Pediatric Care. Clinical Pediatric Emergency Medicine. 


\begin{abstract}
Many hospitals and emergency departments lack resources to optimally care for ill and injured children, perpetuating risks of receiving fragmented and "uneven" care. In this article, we describe the present state of our pediatric emergency medicine workforce as well as the impact that different innovations could have on the future of pediatric emergency care. Many innovative initiatives, including physician and advanced practice provider education and training, pediatric readiness recognition programs, telemedicine and in-situ simulation outreach, and community paramedicine are being utilized to help bridge access gaps and augment the reach of the pediatric emergency medicine workforce. Advocacy for reimbursement for novel care delivery models, such as community paramedicine and telemedicine, and funding for outreach education programming is essential. Also, better understanding of our current training models for and utilization of advanced practice practitioners in pediatric emergency medicine is crucial to understanding the diversity of workforce growth and opportunity.
\end{abstract}

Key words: pediatric emergency medicine, facility recognition, systemization of care, pediatric readiness, telemedicine, community paramedicine, simulation 
In 2006, the Institute of Medicine (IOM) released “Emergency Care for Children: Growing Pains," a treatise on the state of emergency care for children in the United States, noting, "If there is one word to describe pediatric emergency care in 2006, it is uneven". ${ }^{1}$ Seven years later, the Emergency Medical Services for Children (EMSC) program, in partnership with the American College of Emergency Physicians (ACEP), the American Academy of Pediatrics (AAP) and the Emergency Nurses Association (ENA) launched the National Pediatric Readiness Project (NPRP) in an effort to better characterize readiness to care for children in emergency departments (EDs) across the country. Their findings, while noting substantial improvement over prior benchmarking efforts, echoed those of the IOM report. ${ }^{2}$ Despite monumental strides forward and innovative responses to barriers such as access to care; pediatric emergency care in the US remains fragmented and "uneven," with many facilities and regions lacking the resources to optimally care for ill and injured children.2,3

Currently, children under the age of 18 comprise $22.8 \%$ of our country's almost 326 million citizens. ${ }^{4}$ Each year children account for almost 30 million US ED visits. ${ }^{5}$ Though the majority of these pediatric emergency visits occur in general EDs, typically staffed by emergency medicine (EM) physicians, most general EDs care for fewer than 14 children a day. ${ }^{2}$ Geographical challenges also contribute to barriers to optimal care delivery. ${ }^{3,6}$ Comparatively, lower volume EDs, which are more likely to be found in rural or geographically isolated areas, are less prepared to care for children than those who see a higher volume of pediatric patients. ${ }^{2,7}$ EDs in smaller 
hospitals as well as EDs in some larger community hospitals are increasingly forced to rely on larger centers that may be significant distances away. ${ }^{8}$ In fact, at least $29 \%$ of hospitals with EDs either do not admit children or do not have independent pediatric wards with recent studies demonstrating that this number is on the rise. 9 ,

Many innovative initiatives, including systemization of care, telemedicine, in-situ simulation outreach, and community paramedicine are being utilized to help bridge these access gaps and augment the reach of the pediatric emergency medicine workforce. In the following we will describe the present state of our pediatric emergency medicine workforce, as well as the deployment and impact of the mentioned innovations on pediatric emergency care.

\section{OVERVIEW OF THE CURRENT WORKFORCE}

There are several pathways to the practice of Pediatric Emergency Medicine in the US. In 1988, the ACGME defined a new 5-year combined emergency medicine and pediatrics (Emergency Medicine/Pediatrics) training pathway. In 1992 the American Board of Medical Specialties approved subspecialty board certification in Pediatric Emergency Medicine (PEM), dually supported by the American Board of Emergency Medicine (ABEM) and the American Board of Pediatrics (ABP). Currently there are 2,150 physicians board certified in Pediatric Emergency Medicine. Twohundred sixty five of these physicians are certified through the categorical EM 
pathway and 1,885 through the categorical pediatric pathway (11). In addition, there is a cadre of physicians dual certified in categorical emergency medicine and categorical pediatrics. Many of these physicians completed the combined emergency medicine and pediatric residency training programs, while others completed these residencies successively.

The fellowship trained PEM subspecialty workforce practices primarily in freestanding children's hospitals or dedicated pediatric units within general EDs. ${ }^{12}$ In fact, previous workforce studies have shown that only $3 \%$ of fellowship trained PEM boarded physicians work in rural areas. ${ }^{13}$ With respect to the dual trained cohort, a recent survey of combined residency graduates reported that 38\% work at teaching hospitals, while $40 \%$ work at community hospitals. Further $40 \%$ work in general EDs, with an additional $26 \%$ working in free-standing children's hospitals. Geographically, the majority of providers work in urban sites (40\%). Similarly to those with PEM subspecialty fellowship training, only $4 \%$ with dual training practice in rural facilities. ${ }^{14}$ There are currently 4 of these combined emergencymedicine/pediatrics residency training programs nationally. By comparison, there are now 78 PEM fellowship programs in the US and Canada. ${ }^{15}$

This well-trained PEM fellowship and dual trained workforce is a critical component of the nation's pediatric emergency medicine care framework. However, the vast majority of children are initially cared for in general community EDs, where these specialists are less likely to be found. Though EM became a board certified specialty 
in 1979, there remain large pockets of EDs staffed by non-board certified EM physicians, most notably in rural areas. Most often these positions are filled by Internal Medicine (IM) or Family Practice (FP) physicians. ${ }^{1}$ EM physicians are trained to care for children along the entire spectrum of age and illness. However, even highly trained EM physicians may suffer from attrition in skills and decreased confidence in their pediatric knowledge base when faced with low volume exposure to sick children.1,2 By contrast, IM physicians are not trained to care for children at all. Like EM physicians, FP trained physicians are trained to care for patients along the entire age spectrum, however, the focus of this training is preventative and primary care, as opposed to critical illness and injury management. In 2006, experts estimated that almost $40 \%$ of EDs were staffed by non-EM trained physicians. Today, those estimates are closer to $25 \%{ }^{1,16}$ However, board certified emergency medicine coverage in rural areas remains more limited. ${ }^{16}$ As previously noted, access to PEM fellowship or dual trained physicians is also particularly limited in rural areas.

A 2007 AAP policy statement on "Access to Optimal Emergency Care for Children," lauded the development of combined training programs, the increase in PEM fellowship programs, and the increased focus on pediatric training within EM residency programs in response to these workforce issues. At the same time, the AAP called for more subspecialty access, improvement in interfacility transfer processes and more aggressive development and utilization of telemedicine modalities. ${ }^{17}$ Others have noted that combined residency training is also an 
important resource in bridging coverage gaps, particularly in community emergency departments. ${ }^{18}$

Over the last couple of decades, nurse practitioners (NPs) and physician assistants (PAs) have emerged as an important addition to the emergency medicine workforce. Today, more than half of EDs in the country report staffing with these advanced practice providers (APPs). ${ }^{19}$ Currently, there are at least 12 emergency medicine post-graduate programs for APPs. Additionally, there are a handful of graduate-level Emergency Nurse Practitioner (ENP) programs. ${ }^{20}$ There are two board-certifying bodies for NPs. From 2013-2017, the American Nursing Credentialing Center (ANCC) offered a practice pathway for ENP certification. Though this certification pathway is no longer available, 124 nurses achieved ENP certification during this period. ${ }^{19}$ In 2017, the American Academy of Nurse Practitioners Certification Board (AANPCB) introduced a new Emergency NP Board Certification as a specialty certification for certified Family Practice NPs (FNPs) with emergency care expertise. NPs may enter this certification through either practice or training pathways. ${ }^{21}$ As of April 2018, 257 nurses were certified as ENP by the AANPCB. ${ }^{19}$ Nationally, most NPs working in EDs completed training in either FNP or an Acute Care NP programs. ${ }^{1}$

While understanding of the APP utilization in emergency medicine is growing, very little has been described about APP training and practice in pediatric EM. ${ }^{22,23}$ Overall, approximately 6\% of NPs work in EDs or urgent care sites. While we know that $10 \%$ of PAs work in EDs on a national level, little is known about the percentage 
working in pediatric EDs. ${ }^{19}$ In a 2010 study of PA practice patterns $0.12 \%$ were working in PEM. ${ }^{23}$ One reason for this small number may be the limited opportunities for subspecialty training for APPs. In contrast to the number of APP advanced training programs in EM, there are almost no formal training opportunities for PEM. For example, of the 74 programs listed by the Association for Postgraduate Physician Assistant Programs, only one specializes in Pediatric Emergency Medicine. ${ }^{24}$ Many APPs utilize job or facility specific training or continuing education to develop pediatric emergency medicine skills. ${ }^{1}$ Better understanding of the current scope of practice and staffing patterns for APPs within pediatric emergency medicine is important for future workforce planning and solutions.

A heterogeneous workforce provides emergency care in the US. Inherent in this heterogeneity are clear gaps in availability of pediatric emergency medicine providers and training. Though there are many factors contributing to issues with rural and smaller community staffing, there is a substantial challenge in maintaining pediatric subspecialty positions without a large enough patient population to support them. ${ }^{6}$ Innovative strategies are needed to address these and other gaps in subspecialty and dual specialty accessibility and to integrate the resources and expertise of our existing PEM workforce into a wider swath of our nation's emergency medical care. Systemization of care has been proposed as one such strategy to address subspecialty care access. 


\section{SYSTEMIZATION OF CARE}

The Institute of Medicine and EMSC have both called for regionalized systems of care to help fill gaps in local care capacity for children and to improve health outcomes. ${ }^{1,9}$ There are many proposed benefits of systemization of care. Fiscally, maintenance of resources and personnel for low frequency events can be financially prohibitive for smaller hospitals or rural sites. Systemization helps mitigate the impact of these financial stressors. ${ }^{10}$ More importantly, proponents argue that systemization may improve outcomes for high risk, critically ill and injured children by increasing access to optimal care delivery in facilities with high volume experience and a full complement of support services and subspecialists. However, to be successful, this accessibility must be balanced with maintenance of appropriate local resources.

In several recent studies examining the effects of systemization of care, investigators have demonstrated attrition of frontline hospital capacity to care for children with even common pediatric illnesses.$^{10,25}$ In a longitudinal study of transfer practices in California, Massachusetts, Florida and New York, investigators found that the number of facilities providing inpatient care to children in each state substantially decreased, at the same time the number of pediatric transfers increased by $24.6 \%$. Compared to adult care capacity, there was a far greater decline in local availability of pediatric services during this time period. 
Regionalization Indices (RI) (a numerical measure of the degree to which a specific condition is regionalized in care delivery) and Hospital Capability Indices (HCI) (a numerical measure of hospitals' capabilities to care for a broad range of conditions) were trended over a 7-year period. ${ }^{25}$ Overall, HCIs decreased an average of $4 \%$ a year for pediatric conditions, reflecting a decrease in hospital capability for children. Surprisingly, RI for even common pediatric illnesses such as urinary tract infections, asthma and gastroenteritis, increased during this period along with the expected increases in RI for more complex or specialized conditions such as cystic fibrosis and congenital heart disease. ${ }^{25}$

Similarly, in a study by Li et al, looking at transfer rates to 42 pediatric tertiary sites in the US, more than $40 \%$ of children transferred to tertiary sites were transferred for minor illness and injury. The majority of these children were released home directly from the ED. ${ }^{26}$ National ED data sources also demonstrated a near doubling in transfer rates of children ages 1-17 between 2006 and 2014. ${ }^{10}$ In another large, single center study, $25 \%$ of children transferred were discharged within 12 hours of transfer to the receiving facility, without any "further medical intervention, surgical procedure, or special diagnostic workup". ${ }^{27}$ Studies such as these highlight the complexities of systemization efforts and underscore the importance of judicious use of tertiary care centers ${ }^{27}$ Excessive transfers for non-urgent or common pediatric illness, for example, may result in overcrowding and resultant decrease in capacity at tertiary sites. Similarly, decreased exposure to common pediatric illness 
in smaller frontline community hospitals may contribute to attrition in pediatric skills, not only for the physician but also for the entire hospital team. ${ }^{27,28}$

Pediatric medicine has advanced at an extraordinary pace over the last several decades. Coupled with many of these advances has been an increase in children living with complex, chronic medical conditions. ${ }^{6}$ Additionally, inherent in many of these advances has been increasing sub-specialization in pediatric care. Today, for example, utilization of extracorporeal membrane oxygenation in pediatric ICUs in tertiary care centers is not uncommon. These advances clearly necessitate the development of systems of care that can best match children in need of subspecialty expertise and services with the optimal facility for care. However, systemization of care for pediatrics also presents new hazards in our national ability to care for children. The answer to meeting the needs of our pediatric population likely relies on multiple modalities to support a dual pronged approach: ensuring access to higher levels of care for those that truly need it while at the same time creating program and infrastructure to maintain skills and capacity at the local level. This skill and capacity maintenance would be particularly important towards disaster readiness.

\section{Innovative Responses to Workforce Challenges}

As discussed above, solutions to our workforce challenges rely heavily on leveraging the expertise of our subspecialty trained and dual trained PEM workforce to 
improve the care of and resources for children outside of pediatric academic centers. ${ }^{3,26}$ In addition to increasing the workforce, there are several innovations that have shown promise in improving pediatric emergency care. Among these are outreach and education for current practitioners, pediatric readiness recognition programs, technological support systems, telemedicine, ${ }^{26,28}$ and the development and utilization of community paramedicine programs.

\section{Education and Training Outreach}

Pediatric specific education and training through outreach has recently gained momentum as a powerful mechanism for tertiary centers to help close the practice gap between PEM subspecialists and community emergency care providers. These outreach programs, developed to improve knowledge base and skills competencies, are as diverse as they are prolific.

Simulation has a long history of demonstrating improvement in skills and skill maintenance. Multiple types of programs have been described over the past two decades. These programs utilize a variety of different design structures including both low and high fidelity models. In addition, some programs utilize simulation facilitators from outreach sites, whereas others utilize internal hospital staff trained to conduct simulations by the outreach program..$^{29}$ Regardless of simulation design, many of these programs have demonstrated improvement in provider confidence in 
caring for children; others have more clearly shown improvement in skills selfefficacy, performance and outcomes.

Project CAPE is an in-situ program that was designed to improve comfort with critically ill children presenting at critical access hospitals (CAH). ${ }^{29}$ CAHs see a very low volume of pediatric patients and are geographically located in poorly medically resourced areas. In this pilot study, trainer teams from each facility were taught how to perform regular in-situ pediatric simulations in their own institutions. Investigators demonstrated increased provider self-efficacy with certain pediatric procedures, such as intravenous (IV) catheter placement, and increased provider comfort with pediatric patients generally. Interestingly, there was no improvement in provider comfort with intubation, intraosseous (IO) placement or central line placement during this study. By contrast, in another study, directed at decreasing outcome disparities between rural and urban pediatric trauma patients, improvement was seen in provider comfort with several key procedures including spinal immobilization, infant airway management, and chest tube placement. ${ }^{30}$ In this study, simulation facilitators from a pediatric trauma center performed simulated pediatric cases with teams at outlying community EDs. As with the CAPE study, provider comfort with the resuscitation was measured before and after the intervention. Additionally in this trauma system study, the actual team performances, as measured on a scoring rubric, were evaluated and demonstrated positive results. ${ }^{30}$ 
Another team of investigators paired simulation outreach with on-site pediatric readiness assessments and post-simulation dissemination of on-line resources. ${ }^{31}$ Pediatric readiness is a term used commonly to denote an organization's compliance with nationally recognized guidelines for care of children in emergency departments. Facilitators conducted on-site pediatric readiness assessments with the participating hospital before and after the simulation sessions. The simulation sessions themselves were structured to address common errors in the management of pediatric diabetic ketoacidosis, respiratory failure and supraventricular tachycardia, but also inherent in these scenarios were key readiness issues. For example, teams addressing the resuscitation of an infant with respiratory failure also needed to address patient transfer processes, drug administration and equipment stocking. Pediatric readiness (as measured by the weighted pediatric readiness score defined by the NPRP) improved across all sites after these simulation sessions. The investigators also noted that an additional outcome of this outreach was enhanced communication and collaboration between outlying community centers and the academic center. ${ }^{31}$

Overall, study after study has reported that facilities are both receptive to this kind of outreach training and eager for more of it. ${ }^{29}$ One important aspect of simulation, unlike some other outreach modalities, is that it exercises the work of the team in providing care as opposed to just the individual practitioner. This feature is important, as an effective team approach is a critical component of emergent pediatric care. 
Non-simulation based outreach programs are also being employed successfully in an effort to improve pediatric capabilities at local centers. Most notably, there are several on-going efforts as part of the National Pediatric Readiness Project (NPRP). In 2001 the AAP, ACEP and other invested professional organizations developed the first "Guidelines for Care of Children in Emergency Departments." Revised again in 2009, this consensus document, which serves as the foundation for the NPRP, identifies components that are essential to ensuring that an ED is prepared to care for children. Several studies have evaluated ED fidelity to these standards and have found continued gaps in readiness. ${ }^{2,32}$ As part of the NPRP, several programs have been launched in an effort to close the gaps identified. In January of 2018, the EMSC Innovation and Improvement Center (EIIC) launched a 2-year Pediatric Readiness Quality Collaborative designed to help local hospitals institute pediatric specific quality improvement programs. This collaborative is comprised of sixteen teams of training and affiliate sites throughout the country. Utilizing a hub and spoke model, affiliate sites, with mentorship from their training site, as well as collaboration with the other sites nationally, and support from the EIIC, will tackle specific quality improvement bundles for their facility. These bundles include issues like developing disaster plans inclusive of pediatric needs and weighing and documenting children's weight in kilograms. ${ }^{33}$

An essential benefit of all of these outreach programs is an improvement in the connection of tertiary referral centers with outlying facilities. These relationships, 
may improve communication between local care providers and subspecialty providers allowing for more fluid knowledge and resource sharing. ${ }^{30}$

A natural offshoot of the outreach directed towards improving pediatric readiness is a facility recognition program. These programs aim to identify facilities that have met the core requirements outlined in the policy statement on care for children in EDs.

\section{Facility Recognition}

Statewide pediatric readiness recognition programs have been in existence for decades and are present in more than 11 states. ${ }^{32}$ In December of 2017, the EIIC, completed a quality improvement collaborative with 14 additional states to facilitate the development of state-wide pediatric readiness recognition programs. There is significant state-to-state variation in recognition programs. For example some states offer several designation levels for pediatric readiness, while others offer a single designation. Although the majority of states offer voluntary recognition, some states have mandatory programs. Despite the variation in the nuances of recognition levels and certification processes, there is very little variation between states in the fundamental requirements for recognition. These requirements are based on the 2009 consensus document, "Guidelines for Care of Children in Emergency Departments" and include components such as the presence 
of a pediatric emergency care coordinator (PECC), specific QI policies, pediatric transfer guidelines and presence of essential equipment and medications. ${ }^{34,35}$

Underscoring efforts towards pediatric readiness recognition programs is the assumption that these resources and measures will improve pediatric care and outcomes for patients treated at these centers. Although both trauma centers and neonatal intensive care unit designation programs have been associated with improved patient outcomes, there is emerging literature specifically describing the impact of pediatric readiness recognition on pediatric outcomes.

One recent study demonstrated a positive association between facility recognition and pediatric readiness. In a study of pediatric readiness in California, investigators found that facilities designated as pediatric verified through a state facility recognition program had significantly higher pediatric readiness scores than those facilities that were not verified. ${ }^{32}$

In another study, investigators in Arizona, examined pediatric mortality rates before and after hospitals completed verification in a pediatric readiness facility recognition program. Nearly half of Arizona's hospitals have been successfully verified through this process. Overall, there was a trend towards decreased mortality after verification. Although this study had several limitations, it is one of few to associate improved outcomes with the facility recognition process. ${ }^{36}$ 
This early work suggests that facility recognition programs play an important role in addressing gaps in our pediatric care access..$^{35}$ Other innovators have leveraged technology support and platforms as resources to improve care capability at the local level.

\section{TECHNOLOGY SUPPORT SYSTEMS}

Practice changes may significantly lag behind new research at non-academic facilities. A nationally funded Canadian program called TREKK (Translating Emergency Knowledge for Kids) marries cutting edge knowledge translation with a user-friendly on-line platform specifically to address these delays. The TREKK program offers real time best practices with "bottom line" recommendations as well as more comprehensive evidence based reviews, repositories, and guidelines. These resources, free to the user, are also available on a smart phone application. ${ }^{37}$

Another example of technology support addresses medication-dosing error in pediatric care. The Handtevy@C program works with individual EMS agencies and hospitals to create site-specific customized pediatric dosing platforms. Handtevy(C uses technology to limit the need for real time calculations during pediatric resuscitations. Hospital specific formularies are translated so that the correct dosing and volume for administration is immediately provided through a computerized interface for any given weight child. Pediatric equipment sizing is also pre-calculated. ${ }^{38}$ While there is not currently published data demonstrating 
impact in the hospital arena, the Handtevy(c) system has been shown to reduce medication error in pre-hospital care simulations. ${ }^{39}$

Other innovations utilize technology to link outside providers with tertiary care centers. In 2003, the Project Extension for Community Healthcare Outcomes (Project ECHO,) was developed to improve management of hepatitis $\mathrm{C}$ infection by rural primary care physicians in New Mexico. Since that time, this program has been replicated to meet the needs of multiple care communities. In pediatrics, Project ECHO has been adopted by the AAP to improve local care for chronic conditions like epilepsy.

Fundamentally, Project ECHO utilizes tele-education in a hub and spoke model to bring regular, real time education to primary care physicians. Unlike telemedicine, the platform is not designed for specific case consultation, but rather to enhance primary care provider understanding and delivery of up-to-date evidence-based care for specific conditions. Participants in project ECHO take part in weekly teleeducation sessions that include both didactic and case conference components. The initial program in New Mexico improved patient outcomes in the primary care setting, such that patients treated by primary care providers participating in the ECHO program had the same health outcomes as patients treated by specialists. ${ }^{40}$

Tele-education, as a tool, allows for broader access to the expertise provided by academic health centers. While Project ECHO is centered on improving capacity of 
the primary care provider, there may be a role for expanding this type of technology to enhance capacity of rural and community emergency physicians as well.

Telemedicine, while distinct from tele-education, similarly utilizes technology to improve remote access to subspecialty expertise.

\section{TELEMEDICINE}

As previously noted, most pediatric patients are not evaluated in hospitals with easily available access to subspecialty pediatric care. In order to address the needs of children who require pediatric subspecialty care, many programs are bringing the expertise of pediatric subspecialists to the patient via telemedicine. Telemedicine incorporates phone conversations, audiovisual evaluation and communication, as well as echocardiogram and other radiologic services. This novel approach to improving the care of children with minimal increased burden to the workforce leverages technology to aid in the management of children located remotely without access to specialists. There is a significant growing body of evidence supporting the use of telemedicine in pediatrics. In 2015, the American Academy of Pediatrics released a policy statement in support of developing and utilizing telemedicine in the care of pediatric patients. ${ }^{41}$ Its applications are broad in scope including the provision of consultative subspecialty services for community EDs and transport teams, which, in turn, has affected interfacility transfers.

Telemedicine has the potential to effect many domains of care in community EDs.

Kim et al recently published a study evaluating the perception of providers in 
general EDs on pediatric emergency telemedicine. They identified 7 potential uses for telemedicine in the general ED based on physician interviews including: guiding pediatric differential diagnosis; visual diagnosis; alleviating provider fears; guidance on low-frequency high risk events; assessing level of illness; determining disposition; and access to subspecialty consultation. ${ }^{42}$ Other researchers have studied some of the potential uses identified by Kim et al. For instance, multiple studies have identified telemedicine to be a reliable tool for the assessment of severity of respiratory distress in pediatric patients. ${ }^{43,44}$ The ability to assess patients in outside hospitals via pediatric critical care telemedicine and assist in the care of patients prior to their arrival leads to high parent and provider satisfaction, high quality of care, fewer medication errors, a reduction in overall transfers, and less ill children at the time of arrival to a receiving hospital's PICU when compared to children that are not assessed via telemedicine. ${ }^{45-48}$

Telemedicine use in transport medicine occurs regularly in the way of online medical control, yet some transport teams have begun to use audiovisual telemedicine prior to transport. Frequently, transport teams discuss patient care with on-line medical control prior to leaving referral hospitals or while en route. In a recent study, transport team telemedicine had a significant influence on the disposition of patients at the receiving hospital with reduction in ED utilization and increase in direct to PICU admissions. ${ }^{49}$ Use of an Apple-based product for teleconferencing with medical control instead of traditional telemedicine platforms 
was felt to improve assessment and disposition while saving on cost of the platform..$^{50}$

Pediatric telemedicine has a significant and positive effect on the timeliness and need for interfacility transfers. Telemedicine influence on the need for transfer to tertiary care centers is especially evident in rural communities. Telemedicine reduces the need for interfacility transfer from both rural communities as well as community hospitals with hospitalist programs . ${ }^{47,48,51-53}$ This positive affect has significant influence on the feasibility of systemization of care for pediatric patients as well as stress of the limited subspecialty pediatric workforce.

While the beneficial aspects of pediatric telemedicine can be touted, there remain significant barriers to implementing and maintaining such programs. Uscher-Pines and Kahn queried existing, former and future pediatric telemedicine programs to identify such barriers for the success of telemedicine programs . ${ }^{54}$ They identified 6 barriers to pediatric telemedicine programs: credentialing; integration into established workflows; lack of physician buy-in; misaligned incentives; lack of reimbursement; and usability of technology. They recognized that credentialing seems to be one of the largest barriers to provide telemedicine care to outlying hospitals. While some federal regulations regarding credentialing have loosened to allow credentialing by proxy, this continues to be a significant hurdle to begin providing telemedicine consultation. They also note the importance of physician 
buy-in both on the community hospital as well as the hospital providing the consultative services. ${ }^{54}$

Telemedicine has shown itself to be an innovative tool to improve the care of children outside of facilities with pediatric subspecialty care. Not only does it help to ease stress on the workforce but the management provided by such programs significantly improves the health of children in our community.

\section{COMMUNITY PARAMEDICINE}

The role of community paramedicine (CP) and mobile integrated health care (MIHC) continue to expand and create a promising solution for issues involving both pediatric transfers and pediatric workforce. CP and MIHC are programs designed to address the overall wellness of the patients they service. This includes programs in support of preventative services, care for patients with chronic illness, patients determined to be high utilizers, care for patients after being discharged from the hospital, and to develop social support networks. ${ }^{55-59}$

Most CP/MIHC programs in the United States were born out of the need to provide improved access to care for individuals who lived in the rural community. The Red River program is commonly cited as the first CP program in the United States. It was started in 1992 in New Mexico to help improve the medical care for individuals living in the town of Red River (60 miles from the closest practitioner). The 
program educated EMS workers on chronic illness surveillance, education and prevention. The scope of practice for these EMS workers was expanded to provide prescriptions for medications and perform simple procedure in the home. Through the efforts of this revolutionary program, out-of-town transports were reduced by more than 50\%. The Red River program set the baseline for which all subsequent programs have been based. ${ }^{60}$

While the Red River program was not able to sustain itself, there have been many subsequent CP/MIHC programs developed in the United States. The vision of these programs has expanded to not only include rural populations, but also urban populations. They include many different at-risk populations from high ED utilizers to pediatric patients.

Regardless of the focus of the CP/MIHC program, the success of the program rests on advanced training for the CP provider. Programs have approached education of the providers in many different ways. Some programs have utilized formal CP education associated with colleges or paramedic programs. North Central EMS institute was among the first in the United States to develop such a program that involved classroom learning as well as clinical time to learn the expanded skills necessary to become a CP provider ${ }^{56}$ Still other programs have depended on homegrown curriculum designed specifically to address the skills and knowledge to care for the population being served. In Indianapolis, a training program was designed and implemented to educate community paramedics on pediatric asthma 
intervention. The curriculum involved classroom education on asthma, asthma treatment and disease recognition as well as time spent in an asthma clinic with a pulmonologist and in a pediatric intensive care unit.

There is significant emerging evidence regarding CP/MIHC implementation among adult populations in terms of resource utilization, specific disease intervention, referral success, and improving the medical home. Concurrently, there is a paucity of literature regarding the utilization of $\mathrm{CP} / \mathrm{MIHC}$ programs in pediatric patients. The Treat the Streets program developed in Indianapolis, IN is one example of a pediatric specific $\mathrm{CP} / \mathrm{MIHC}$ program.. The program leveraged the skills of $\mathrm{CP}$ providers after focused intensive training, as previously mentioned, to decrease hospital recidivism rates among children with acute asthma exacerbations. While the data is not published, personal correspondence with the investigators revealed a decrease in admission recidivism during the study period without a statistically significant change in recidivism in the study group when compared to the control group. Importantly, the study did show that implementation of a CP program can be successful in the pediatric population.

$\mathrm{CP}$ and MIHC are emerging programs that help to address many of the workforce issues facing emergency medicine as whole. Demonstrating effectiveness among pediatric populations is sparse in the literature, yet extrapolation among adult literature is promising for pediatric CP interventions. 


\section{SUMMARY}

Over the last several decades, efforts to improve access to appropriate pediatric emergency care services have focused primarily on two approaches: 1) developing the workforce; and 2) developing innovations to support and amplify the impact of this workforce outside of pediatric specialty centers. Although many of these innovations show promise, there is a need for more rigorous evaluation of the effect of these new programs both on patient outcomes and provider development. Additionally, advocacy for reimbursement for novel care delivery models, such as community paramedicine, and funding for outreach education programming is essential. Finally, better understanding of our current training models for and utilization of APPs in PEM is crucial to understanding the diversity of workforce growth and opportunity. The scope and structure of our pediatric emergency medicine workforce will continue to evolve to meet the needs of our ever-changing healthcare landscape. This evolution must include deliberate strategies that bolster local community readiness for the care of children. National and state infrastructures should be nimble and prepared to support shifts in workforce development to meet the needs of local, regional and national community readiness. 


\section{References}

1. Institute of Medicine (US) Committee on the Future of Emergency Care in the United States Health System. Emergency care for children: Growing pains. Washington, DC: National Academies Press, 2007.

2. Gausche-Hill M, Ely M, Schmuhl P, et al. A national assessment of pediatric readiness of emergency departments. JAMA Pediatr 2015; 169:527-34.

3. Mayer ML. Disparities in geographic access to pediatric subspecialty care. Matern Child Health J 2008; 12:624-32.

4. United States Census Bureau QuickFacts. Available at: https://www.census.gov/quickfacts/fact/table/US\#viewtop. Accessed $7 / 28 / 2018$.

5. Centers for Disease Control and Prevention. FastFacts - National Center for Health Statistics - Emergency Department Visits. Available at: https://www.cdc.gov/nchs/fastats/emergency-department.htm. Accessed $7 / 28 / 2018$.

6. Basco WT, Rimsza ME, American Academy of Pediatrics Committee on Pediatric Workforce. Pediatrician workforce policy statement. Pediatrics 2013; 132:390-7.

7. Ray KN, Olson LM, Edgerton EA, et al. Access to high pediatric-readiness emergency care in the United States. J Pediatr 2018; 194:225-32.

8. Chamberlain JM, Krug SE, Shaw KN. Emergency care for children in the United States. Health Aff (Millwood) 2013; 32:2109-15.

9. Horeczko T, Marcin JP, Kahn JM, et al. Urban and rural patterns in emergent pediatric transfer: a call for regionalization. J Rural Health 2014; 30:252-8.

10. Franca UL, McManus ML. Availability of definitive hospital care for children. JAMA Pediatr 2017; 171: e171096.

11. ABMS Board Certification Report 2016-17. Available at: http://www.abms.org/media/139572/abms board certification report 201 6-17.pdf. Accessed 7/28/2018.

12. Gorelick MH, Schremmer R, Ruch-Ross H, et al. Current workforce characteristics and burnout in pediatric emergency medicine. Acad Emerg Med 2016; 23:48-54.

13. Althouse LA, Stockman JA, 3rd. Pediatric workforce: a look at pediatric emergency medicine data from the American Board of Pediatrics. J Pediatr 2006; 149:600-2.

14. Strobel AM, Chasm RM, Woolridge DP. a survey of graduates of combined emergency medicine-pediatrics residency programs: an update. J Emerg Med 2016; 51:418-25.

15. American Medical Association. Residency and fellowship database. Available at: https://www.ama-assn.org/search/amaassn/residency\%20and\%20fellowship\%20database. Accessed 7/2/2018.

16. Reiter M, Wen LS, Allen BW. The emergency medicine workforce: profile and projections. J Emerg Med 2016; 50:690-3. 
17. Yamamoto LG, American Academy of Pediatrics Committee on Pediatric Emergency Medicine. Access to optimal emergency care for children. Pediatrics 2007; 119:161-4.

18. Walthall J. Thinking and training together for pediatric emergency care. J Emerg Med 2016; 51:192-3.

19. Evans D, Wilbeck J. Emergency nurse practitioner practice data. Available at: http://aaenpnatl.org/images/articles/ENPPractice Data Exec Summary FINAL042518.p df. Accessed 7/28/2018.

20. American Academy of Emergency Nurse Practitioners. NP fellowship programs. Available at: https://aaenpnatl.org/content.php?page=Fellowship_Programs. Accessed 7/28/18.

21. American Academy of Nurse Practitioners Cerfication Board. Certification process. Available at: www.aanpcert.org/certs/process. Accessed 7/28/2018.

22. Freed GL, Dunham KM, Loveland-Cherry CJ, et al. Pediatric nurse practitioners in the United States: current distribution and recent trends in training. J Pediatr 2010; 157:589-93.

23. Freed GL, Dunham KM, Moote MJ, et al. Pediatric physician assistants: distribution and scope of practice. Pediatrics 2010; 126:851-5.

24. The Association of Postgraduate PA Programs. Post-graduate PA programs. Available at: http://appap.org/nemoursalfred-dupont-hospital-childrenpediatric-emergency-medicine-physician-assistant-fellowship/. Accessed 7/28/2018.

25. Franca UL, McManus ML. Trends in regionalization of hospital care for common pediatric conditions. Pediatrics 2017; e20171940; DOI: 10.1542/peds.2017-1940.

26. Li J, Monuteaux MC, Bachur RG. Interfacility transfers of noncritically ill children to academic pediatric emergency departments. Pediatrics 2012; 130:83-92.

27. Gattu RK, Teshome G, Cai L, et al. Interhospital pediatric patient transfersfactors influencing rapid disposition after transfer. Pediatr Emerg Care 2014; 30:26-30.

28. Carr BG, Edwards JM, Martinez R. Regionalized care for time-critical conditions: lessons learned from existing networks. Acad Emerg Med 2010; 17:1354-8.

29. Katznelson JH, Mills WA, Forsythe CS, et al. Project CAPE: a high-fidelity, in situ simulation program to increase critical access hospital emergency department provider comfort with seriously ill pediatric patients. Pediatr Emerg Care 2014; 30:397-402.

30. Bayouth L, Ashley S, Brady J, et al. An in-situ simulation-based educational outreach project for pediatric trauma care in a rural trauma system. J Pediatr Surg 2018; 53:367-71.

31. Abulebda K, Lutfi R, Whitfill T, et al. A collaborative in situ simulation-based pediatric readiness improvement program for community emergency departments. Acad Emerg Med 2018; 25:177-85. 
32. Remick K, Kaji AH, Olson L, et al. Pediatric readiness and facility verification. Ann Emerg Med 2016; 67:320-8.

33. EMSC Innovation and Improvement Center. Pediatric readiness quality collaborative. Available at: https://emscimprovement.center/collaboratives/PRQuality-collaborative/. Accessed 7/28/2018.

34. Gausche-Hill M, Krug SE, American Academy og Pediatrics Committee on Pediatric Emergency Medicine, American College of Emergency Physicians Pediatric Committee, Emergency Nurses Association Pediatric Committee. Joint policy statement - guidelines for care of children in the emergency department. Pediatrics 2009; 124:1233-43.

35. Barata IA, Stadnyck JM, Akerman M, et al. Novel approach to emergency departments' pediatric readiness across a health system. Pediatr Emerg Care 2018; Feb 5. doi: 10.1097/PEC.0000000000001385. [Epub ahead of print]

36. Rice A, Dudek J, Gross T, et al. The impact of a pediatric emergency department facility verification system on pediatric mortality rates in Arizona. J Emerg Med 2017; 52:894-901.

37. TREKK. Translating emergency knowledge for kids. Available at: https://trekk.ca. Accessed 7/28/2018.

38. Handtevy Pediatric Emergency Standards. Available at: https://www.handtevy.com/solutions/hospital/. Accessed 7/28/2018.

39. Rappaport LD, Brou L, Givens T, et al. Comparison of errors using two lengthbased tape systems for prehospital care in children. Prehosp Emerg Care 2016; 20:508-17.

40. Zhou C, Crawford A, Serhal E, et al. The impact of Project ECHO on participant and patient outcomes: a systematic review. Acad Med 2016; 91:1439-61.

41. Marcin JP, Rimsza ME, American Academy of Pediatrics Committee on Pediatric Workforce. The use of telemedicine to address access and physician workforce shortages. Pediatrics 2015; 136: 202-9.

42. Kim JW, Tiyyagura G, Langhan M. A qualitative analysis of general emergency medicine providers' perceptions on pediatric emergency telemedicine. Pediatr Emerg Care 2017; Feb 21. doi: 10.1097/PEC.0000000000001067. [Epub ahead of print]

43. Siew L, Hsiao A, McCarthy P, et al. Reliability of telemedicine in the assessment of seriously ill children. Pediatrics 2016; 137:e20150712.

44. Gattu R, Scollan J, DeSouza A, et al. Telemedicine: a reliable tool to assess the severity of respiratory distress in children. Hosp Pediatr 2016; 6:476-82.

45. Dayal P, Hojman NM, Kissee JL, et al. Impact of telemedicine on severity of illness and outcomes among children transferred from referring emergency departments to a children's hospital PICU. Pediatr Crit Care Med 2016; 17: 516-21.

46. Dharmar M, Kuppermann N, Romano PS, et al. Telemedicine consultations and medication errors in rural emergency departments. Pediatrics 2013; 132:1090-7. 
47. Dharmar M, Romano PS, Kuppermann N, et al. Impact of critical care telemedicine consultations on children in rural emergency departments. Crit Care Med 2013; 41:2388-95.

48. Yang NH, Dharmar M, Kuppermann N, et al. Appropriateness of disposition following telemedicine consultations in rural emergency departments. Pediatr Crit Care Med 2015; 16:e59-64.

49. Fugok K, Slamon NB. The effect of telemedicine on resource utilization and hospital disposition in critically ill pediatric transport patients. Telemed J E Health 2018; 24:367-74.

50. Patel S, Hertzog JH, Penfil S, et al. A prospective pilot study of the use of telemedicine during pediatric transport: a high-quality, low-cost alternative to conventional telemedicine systems. Pediatr Emerg Care 2015; 31:611-5.

51. Labarbera JM, Ellenby MS, Bouressa P, et al. The impact of telemedicine intensivist support and a pediatric hospitalist program on a community hospital. Telemed J E Health 2013; 19:760-6.

52. Holt T, Sari N, Hansen G, et al. Remote presence robotic technology reduces need for pediatric interfacility transportation from an isolated northern community. Telemed J E Health 2018; Feb 2. doi: 10.1089/tmj.2017.0211. [Epub ahead of print]

53. Webb CL, Waugh CL, Grigsby J, et al. Impact of telemedicine on hospital transport, length of stay, and medical outcomes in infants with suspected heart disease: a multicenter study. J Am Soc Echocardiogr 2013; 26:1090-8.

54. Uscher-Pines L, Kahn JM. Barriers and facilitators to pediatric emergency telemedicine in the United States. Telemed J E Health 2014; 20:990-6.

55. Huang YH, Ma L, Sabljak LA, et al. Development of sustainable community paramedicine programmes: a case study in Pennsylvania. Emerg Med J 2018; 35:372-8.

56. Bigham BL, Kennedy SM, Drennan I, et al. Expanding paramedic scope of practice in the community: a systematic review of the literature. Prehosp Emerg Care 2013; 17:361-72.

57. Glenn M, Zoph 0 , Weidenaar K, et al. State regulation of community paramedicine programs: a national analysis. Prehosp Emerg Care 2018; 22: 244-51.

58. American College of Emergency Physicians. Medical direction of mobile integrated health care and community paramedicine programs. Policy statement. Ann Emerg Med 2015; 66:692-3.

59. Joint Committee on Rural Emergency Care, National Association of State Emergency Medical Services Officials, National Organization of State Offices of Rural Health. State perspectives discussion paper on development of community paramedic programs. Available at: https://www.nasemso.org/Projects/RuralEMS/documents/CPDiscussionPa per.pdf. Accessed 7/28/2018.

60. Wingrove G, Laine S. Community paramedic: a new expanded EMS model. Available at:

http://healthandwelfare.idaho.gov/Portals/0/Medical/EMS/NAEMSE Com munity Paramedic Article.pdf. Accessed 7/28/18. 